TEME, г. XLIII, бр. 4, октобар - децембар 2019, стр. 1201-1215

\begin{tabular}{lr}
\hline \hline Оригинални научни рад & https://doi.org/10.22190/TEME191101072S \\
Примљено: 1. 11.2019. & UDK 327.56:351.88(497.11:4-672EU)
\end{tabular}

Ревидирана верзија: 23. 11. 2019.

Одобрено за штампу: 1. 12. 2019.

\title{
BALANCING NATIONAL SECURITY AND COMPETITIVENESS IN THE AGE OF INFORMATION ${ }^{a}$
}

\author{
Jelena Stanojević ${ }^{1 *}$, Miloš Pavlović ${ }^{2}$ \\ ${ }^{1}$ University of Priština, Faculty of Economics, Kosovska Mitrovica, Serbia \\ ${ }^{2}$ Belgrade Business Academy for Applied Studies, Belgrade, Serbia \\ jelena.stanojevic@pr.ac.rs
}

\begin{abstract}
A secure environment plays a crucial role in the overall stability of the economy, having an impact and contributing to its competitiveness. Although an absence of security risks cannot certainly drive the country's economic development and competitiveness, its existence would definitely have far-reaching consequences for the economy. The paper aims to analyze the national security of the European (EU) countries and Serbia, reflected in the following security indicators: business costs of terrorism, business costs of crime and violence, organized crime, and the reliability of policy services. In this context, the study provides a comparative analysis of the mentioned indicators in order to assess their trend, as well as the correlation between national security and national competitiveness with the aim to determine how national security affects national competitiveness. The empirical analysis of the study includes 28 EU member countries and Serbia as a candidate country, covering the period from 2011 to 2017, with data available in the Global Competitiveness Reports. The findings of this study allow for a better understanding of the overall national security and its impact on the national competitiveness in the context of the EU and Serbia.
\end{abstract}

Key words: national security, competitiveness, indicators, European Union, Serbia.

\section{БАЛАНСИРАЊЕ НАЦИОНАЛНЕ БЕЗБЕДНОСТИ И КОНКУРЕНТНОСТИ У ДОБА ИНФОРМАЦИЈА}

\section{Апстракт}

Безбедно окружење игра изузетно важну улогу у одржавању стабилности привреде и тиме битно утиче на њену укупну конкурентност и доприноси јој. Иако одсуство безбедносних ризика не води само по себи економском развоју и конкурентности, њихово постојање би са сигурношћу имало далекосежне последице по

${ }^{a}$ The research is realised under the projects no. 179066 and 47023 that is financed by the Ministry of Education, Science and Technological Development of the Republic of Serbia. 
привреду. Циљ рада је да се анализира национална безбедност европских (ЕУ) земаља и Србије, на основу следећих показатеља безбедности: пословни трошкови проузроковани тероризмом, пословни трошкови проузроковани криминалом и насиљем, организовани криминал и поузданост политичких услуга. Сходно томе, у раду је дата упоредна анализа поменутих показатеља како би се утврдио ниво показатеља у ЕУ и Србији, сагледао тренд у датом временском периоду, као и корелација између националне безбедности и националне конкурентности с циљем утврђивања утицаја који национална безбедност има на националну конкурентност. Емпиријска анализа укључује 28 земаља чланица ЕУ и Србију као државу кандидата, а за период од 2011. до 2017. године. Информациону основу чине подаци доступни у Извештају о глобалној конкурентности. Резултати истраживања пружају могућност бољег разумевања концепта националне безбедности и њеног утицаја на националну конкурентност у контексту ЕУ и Србије.

Кључне речи: национална безбедност, конкурентност, индикатори, Европска унија, Србија.

\section{INTRODUCTION}

Extremely rapid scientific and technological development, diffusion of modern scientific achievements and technology and their increasing impact on all areas of social life, boost the complexity of the global environment. In this context, there will be a greater potential for positive impact on the economic development of countries through the implementation of innovative technological solutions that will contribute to the proper use of resources and accelerate productivity growth. However, contrary to expectations that the responsibility of all involved actors will be increased for the use of scientific advances in the general interest and for the benefit of all humanity, it is estimated that the development of science and technology will continue to be subject to various forms of abuse, leading to negative security implications. The dynamics of global information technology development will further facilitate and intensify different crime activities (National Security Strategy of the Republic of Serbia - draft, 2017, p. 4).

The last decade of XX century and the beginning of XXI century were under the influence of new security trends in the world. These trends shifted from, initially, military to other areas, such as the economy, energy, ecology, but also the security of individuals (civilians), as well as the entire society (National Security Strategy of the Republic of Serbia, 2009, p. 4).

National and international security has become one of the biggest challenges over the last years, and the core objective for national and international organizations. Given the ever-changing security environment and the various perspectives that need to be taken into consideration, the concept of security is not easy to define. In general, security is considered the ability of a country to protect its territory and citizens and respond to all kinds of threats that may evolve and emerge over time (Nikolaevich et al., 2018, p. 462). Given its importance from the aspect of urgency and criticality, special attention should be given to a comprehensive analysis of security issues from different perspectives. 
The circumstances that lead to security risks at the global level are various, and it is the gap in the level of economic and cultural development that leads to poverty and social vulnerability of the population, negative demographics and negative psycho-social phenomena, to name some of them. Regional and local conflicts, ethnical and religious extremism, terrorism, organized crime, arms trade, illegal migration, lack of natural resources, corruption, etc., jeopardize the stability of economies and thus national and international security. The impact of unstable economies that do not provide safe life and business environment can be reflected in various aspects: a decline in investments, the fall in demand, movement of supply of goods and service including financial flow, etc. The common feature to all security risks is their unpredictable, asymmetrical and transnational character (National Security Strategy of the Republic of Serbia, 2009, p. 4).

The list of security problems that can affect the competitiveness of a country is long and diverse. Among them, special attention should be given to those that harm the international position of a country and its competitiveness. Namely, national security problems, reflected in terrorism, crime, violence, etc., have an equal influence on national competitiveness as other micro and macroeconomic determinants. All these security issues become a part of the national competitive business strategy.

The paper explores national competitiveness through the prism of national security in order to assess the correlation between security and competitiveness. The author identifies the national security indicators that are key to national competitiveness and contribute to a country's position in global economy. Namely, these indicators are: business costs of terrorism, business costs of crime and violence, organized crime, and the reliability of policy services, as indicators pertaining to national competitiveness. The subject of the analyses are the EU countries and Serbia in the period from 2011 to 2017.

\section{LITERATURE REVIEW}

Over the last decades and worldwide, many people have benefited from the expansion of the online environment and plenty of economic opportunities have been introduced in this new era. In the information age, only "smart economies" will be competitive on the global market and only these economies will be able to provide a proper nation's defence.

A modern economy cannot be considered the result of actions of million isolated market players, but as a joint result within a framework of national economies (Botos, 2006, p. 13). Improving competitiveness implies not only a well-functioning market, but also strong institutions with the quality of adaptability and capacity for innovation. These essential elements will become even more important in the future, given that competitive economies take risks more easily, and adapt to the rapidly changing 
environment (WEF, 2018, p. 2). Cho and Moon (2001, p. 55) define competitiveness as the country's ability to produce products and provide services that meet the tastes of international competition, while the citizens of that country enjoy a sustainable living standard. In general, competitiveness should ensure a high standard of living, poverty reduction and job opportunities to all (Lang, 2009, p. 26).

One of the key roles in national competitiveness belongs to the state, and this role is reflected in the state's aspiration to constantly stimulate the improvement of the economy and the process of innovation (Stanojević, 2018 , p. 85). Given that some economies are technologically superior, offering innovative and high quality products, etc., the question of what determines competitiveness must be raised. Individual factors and determinants that contribute to the progress of the economy and its competitiveness should be taken into consideration. Institutions (public and private), the infrastructure, the macroeconomic environment, education, the market (goods, labor, financial) efficiency and size, technology, research and development and innovation, are some of the indicators that need to be analyzed (Krstić, Stanojević \& Stanišić, 2016, p. 1038).

Institutions, as one of national competitiveness indicators, can be affected by different security determinants. Threats that jeopardize national security by themselves have a negative impact on national competitiveness. However, strict rules and regulations in order to prevent these threats and protect a nation can also impact competitiveness in a negative manner, impeding the free movement of goods, services, capital and labor (Long, 2013, p. 46).

The concept of security can be elaborated at the individual, national and international level. While individual security is related to the individual impact on security issues, national and international security involves a wide range of national and international organizations and institutions dealing with security risks and trying to prevent, minimize and/or eliminate them (Simanavičienè, Stankevičius, 2015, p. 128).

The traditional concept of national security refers to a nation's intention to protect their territory and natural resources by using military power. Political independence and territory integrity are the values that should be protected. This concept is based on the protection of foreign policy interests in international relations, territories from external aggression, the order of government and governing regimes (in socialist countries), and focusing on the security of people and their participation in international and global security.

In the modern era, instead of the state as a sole "security provider", individuals and non-governmental, subnational and transnational entities take on the role of active security entities. In addition to traditional functions (diplomatic, intelligence and defence), the modern state emphasizes the importance of economic, energy, cultural, environmental, social, information 
and other security aspects (Mijalković, 2009, p. 59). All this indicates a change in the concept of national security which is adapted to the conditions and needs of contemporary security reality. Today's national security implies different kinds of treats that require state protection through diplomacy, economic or political power (Borrus, Zysman, 1990, p. 4). National security is a wide term that refers to different types of securities in addition to the military, such as: monetary, political, economic, energetic, environmental and natural resources.

In "The Concept of Security" (Baldwin (1997, p. 14), it is stated that security is a condition difficult to be qualified since "we shall either be secure, or we shall be insecure. We cannot have partial security. If we are only half secure, we are not secure at all". However, security by itself causes certain costs that could be managed with other purposes in mind. In addition to the costs incurred by providing a certain level of national security, there are costs caused by the already existing crime, terrorism and violence. Accordingly, there are indicators and measures that quantify various security risks. In the paper, security indicators are considered to be those related to national competitiveness, and they are listed in the Global Competitiveness Report as (public) institutional competitiveness.

\section{DATA AND METHODOLOGY}

The aim of the research is to provide an overview and to analyze national security, based on the following indicators: business costs of terrorism, business costs of crime and violence, organized crime, and the reliability of policy services. These four indicators go under the public institutions and belong to the first pillar - Institutions among eleven other pillars that compose the Global Competitiveness Index (GCI). "The institutional environment is determined by the legal and administrative framework within which individuals, firms, and governments interact to generate wealth" (Global Competitiveness Report, 2013, p. 4). The quality of institutions has influence on the overall national competitiveness and economic growth by affecting investment decisions and other economic activities. Explicitly, investors are not willing to invest their capital in case of an insecure environment where there is no efficient protection of rights.

The subject of the analysis are the $28 \mathrm{EU}$ countries and Serbia as a candidate country. The information base of the analysis are the World Economic Forum data available in the Global Competitiveness Reports in the period from 2011 to 2017. The methods used in the analysis are descriptive statistics, correlation, and comparative analysis.

The purpose of the analysis is to examine the trend of the four security indicators that reflect the national security of the EU countries and examine the position of Serbia towards them, as well as examine the inter-correlation between individual indicators in the analyzed period. 
Further, the correlation analysis highlights the relation between national security and competitiveness in order to estimate the influence of security to national competitiveness.

\section{RESULTS AND DISCUSSION}

a) Comparative analysis of national security as an important factor of overall competitiveness of the EU countries and Serbia

National security refers to the overall security of a country and the safety of the environment of its citizens, the economy, and its institutions. In the paper, national security is analyzed in terms of the four indicators that are considered as the main determinants of security and accordingly impact the competitiveness of public institutions and overall institutions.

Table 1 provides an overview of the security indicators (business costs of terrorism, business costs of crime and violence, organized crime, and the reliability of policy services), as well as the Global Competitiveness Index (GCI), and Pillar 1- Institutions as one of the 12 pillars in the GCI. The analyzed period covers the years from 2011 to 2017 for the EU countries and Serbia. The value of indicators ranges between 1 and 7 , where 7 indicates the most desirable outcome.

Based on the data provided in table 1, the highest score of almost all four security indicators in the whole analyzed period is recorded in Finland. Also, Finland records the highest score for the overall competitiveness and the competitiveness of institutions. For some of the security indicators and analyzed years, high scores are also recorded in Germany, Denmark, Luxemburg, Netherlands, Slovenia and Sweden.

As for the lowest score of the security indicators, many countries faced security issues in the analyzed period. Based on the data provided in table 1, Bulgaria is the EU country that suffers the most from the negative security factors. However, other EU countries also record low results for certain security indicators in the analyzed years. Namely, Denmark recorded the lowest score for business costs of terrorism in 2013 and Belgium in 2017. Since 2014, the business costs of terrorism score are the lowest in France.

The score for the Pillar 1 - Institutions is low in Hungary and Croatia, while Italy has the biggest concern regarding organized crime. Other countries with low scores are Romania and Slovakia. Greece is the EU country with the lowest GCI in the entire analyzed period, which is not the case with other the security indicators.

Serbia, as the EU candidate country, shows results pertaining to security indicators that do not deviate from the results of the EU countries. However, for the Pillar 1 in the GCI - Institutions, Serbia records slightly lower results compared to the EU countries. 
Table 1. Security indicators for the EU countries and Serbia in the period 2011-2017

\begin{tabular}{|c|c|c|c|c|c|c|c|c|c|c|c|c|c|c|c|c|c|c|c|c|c|c|c|c|}
\hline \multirow[b]{2}{*}{$\begin{array}{l}\text { Country } \\
\text { Name }\end{array}$} & \multicolumn{6}{|c|}{2011} & \multicolumn{6}{|c|}{2012} & \multicolumn{6}{|c|}{2013} & \multicolumn{6}{|c|}{2014} \\
\hline & 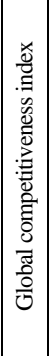 & 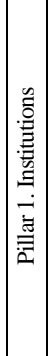 & 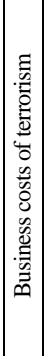 & 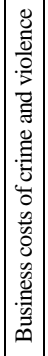 & 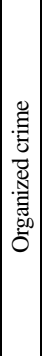 & 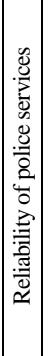 & 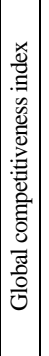 & 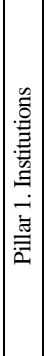 & 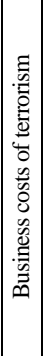 & 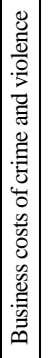 & 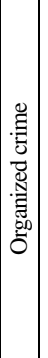 & 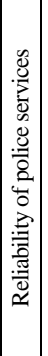 & 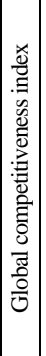 & 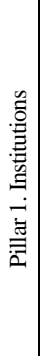 & 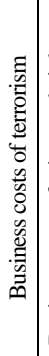 & 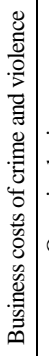 & 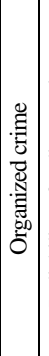 & 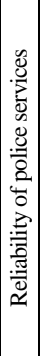 & 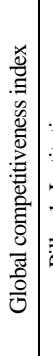 & 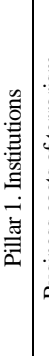 & 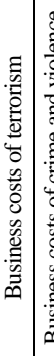 & 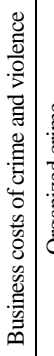 & 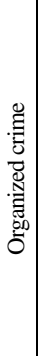 & 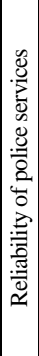 \\
\hline (S) & & 5 & 6.5 & 5.6 & 6.4 & 5.9 & 5.2 & 5.0 & 6.6 & 5.8 & 6.4 & 6.0 & 5.2 & \begin{tabular}{|l|}
5.1 \\
\end{tabular} & 6.6 & 6.06 & 6.5 & 5.9 & \begin{tabular}{|l|l|}
5.2 & 5
\end{tabular} & \begin{tabular}{l|l}
5.1 & 6
\end{tabular} & \begin{tabular}{l|l|l}
6.5 & 6
\end{tabular} & \begin{tabular}{l|l}
6.1 & 6
\end{tabular} & 6.5 & 5.9 \\
\hline elgium & 5.2 & 5.0 & 6.2 & 5.7 & 6.3 & 5.6 & 5.2 & 5.0 & 6.2 & 5.8 & 6.1 & 5.7 & 5.1 & \begin{tabular}{|l|l|}
5.0 \\
\end{tabular} & 6.15 & \begin{tabular}{|l|l}
5.5 & 6 \\
\end{tabular} & \begin{tabular}{|c|c|}
6.0 & $=$
\end{tabular} & 5.6 & \begin{tabular}{|l|l|}
5.2 & 5 \\
\end{tabular} & \begin{tabular}{l|l}
5.1 & 6
\end{tabular} & \begin{tabular}{l|l}
6.0 & 5 \\
\end{tabular} & \begin{tabular}{l|l}
5.5 & 6
\end{tabular} & 6.1 & 5.7 \\
\hline тости & 2 & 3 & 4.9 & 4.0 & 3.9 & 3.4 & \begin{tabular}{|l|}
4.3 \\
\end{tabular} & 3.4 & 4.8 & 3.8 & \begin{tabular}{|l|}
3.9 \\
\end{tabular} & 3.4 & 4.3 & 3.4 & \begin{tabular}{|l|l|}
4.9 & 3
\end{tabular} & \begin{tabular}{|l|l|}
3.9 & 3 \\
\end{tabular} & \begin{tabular}{|l|l}
3.8 \\
\end{tabular} & 3.4 & \begin{tabular}{|l|l|l}
4.43 \\
\end{tabular} & \begin{tabular}{l|l}
3.3 & 5
\end{tabular} & \begin{tabular}{l|l}
5.1 & 4 \\
\end{tabular} & \begin{tabular}{l|l}
4.3 & 4
\end{tabular} & 4.0 & . \\
\hline prus & 4 & 4.8 & \begin{tabular}{|l|}
5.9 \\
\end{tabular} & 5.7 & 5.6 & 5.2 & \begin{tabular}{|l|l|}
4.3 & \\
\end{tabular} & 4.6 & 6.0 & 5.6 & 5.7 & 5.1 & 4.3 & 4.5 & 6.05 & \begin{tabular}{|l|l}
5.5 & 5
\end{tabular} & \begin{tabular}{|l|l}
5.7 \\
\end{tabular} & \begin{tabular}{|l|l|}
4.8 \\
\end{tabular} & \begin{tabular}{|l|l}
4.3 & 4 \\
\end{tabular} & \begin{tabular}{l|l}
4.4 & 6
\end{tabular} & \begin{tabular}{l|l}
6.0 & 5 \\
\end{tabular} & \begin{tabular}{l|l}
5.7 & 5
\end{tabular} & 5.7 & 4.7 \\
\hline ech & 4.5 & 3.6 & 6.4 & 5.5 & 5.5 & 3.6 & 4.5 & 3.7 & 6.5 & 5.4 & \begin{tabular}{|l|}
5.4 \\
\end{tabular} & 3.8 & 4.4 & 3.6 & \begin{tabular}{|l|}
6.45 \\
\end{tabular} & \begin{tabular}{|l|l}
5.0 &
\end{tabular} & \begin{tabular}{|l|l}
5.0 \\
\end{tabular} & \begin{tabular}{|l|l|}
3.9 \\
\end{tabular} & \begin{tabular}{l|l|l}
4.5 & 3 \\
\end{tabular} & \begin{tabular}{l|l}
3.8 & 5
\end{tabular} & \begin{tabular}{l|l}
5.8 & 4 \\
\end{tabular} & \begin{tabular}{l|l}
4.8 & 5
\end{tabular} & 5.0 & 4.1 \\
\hline . & 4 & 5.3 & 5.8 & 5.6 & 5.9 & \begin{tabular}{|l|}
5.9 \\
\end{tabular} & 5.5 & 5.3 & 5.8 & 5.8 & 6.0 & 5. & 5.5 & 5.3 & \begin{tabular}{|l|l|}
5.7 &
\end{tabular} & 5.6 & 86 & 6.0 & \begin{tabular}{|l|l|}
5.5 & 5
\end{tabular} & \begin{tabular}{l|l}
5.2 & 5
\end{tabular} & \begin{tabular}{l|l}
5.5 & 5 \\
\end{tabular} & \begin{tabular}{l|l}
5.2 & 5
\end{tabular} & 5.5 & 5.9 \\
\hline n & & 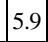 & 6.3 & 6.2 & 6.8 & 6.3 & 5.3 & 5.4 & 5.5 & 5.2 & 6.1 & 6 & 5.2 & \begin{tabular}{|l|l|}
5.2 & \\
\end{tabular} & \begin{tabular}{|l|l|}
4.9 \\
\end{tabular} & 4.65 & 5.50 & 6.1 & 5 & \begin{tabular}{l|l}
5.3 & 4 \\
\end{tabular} & \begin{tabular}{l|l}
4.8 & 4 \\
\end{tabular} & \begin{tabular}{l|l}
4.7 & 5 \\
\end{tabular} & 5.5 & 60 \\
\hline ain & 5 & 4.3 & 5.1 & 5.4 & 5.7 & 5.8 & \begin{tabular}{|l|l}
4.6 \\
\end{tabular} & 4.2 & 5.3 & 5.5 & 5.8 & 6.0 & 4.6 & 4.1 & 5.25 & 5.5 & 5.7 & \begin{tabular}{|l|l|}
5.9 \\
\end{tabular} & \begin{tabular}{|l|l|}
4.6 & 3 \\
\end{tabular} & \begin{tabular}{l|l}
3.8 & 5
\end{tabular} & \begin{tabular}{l|l}
5.0 & 5
\end{tabular} & \begin{tabular}{l|l}
5.2 & 5
\end{tabular} & 5.5 & 5.8 \\
\hline Estonia & 6 & 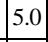 & 6.4 & 5.5 & 6.6 & 5.5 & \begin{tabular}{|l|l}
4.6 \\
\end{tabular} & 4.9 & 6.4 & 5.5 & 6.6 & 5.5 & 4.7 & \begin{tabular}{|l|}
4.9 \\
\end{tabular} & \begin{tabular}{|l|l|}
6.45 \\
\end{tabular} & \begin{tabular}{|l|l}
5.6 & 6 \\
\end{tabular} & 6.45 & \begin{tabular}{|l|l|}
5.3 \\
\end{tabular} & \begin{tabular}{|l|l|}
4.7 & 5 \\
\end{tabular} & \begin{tabular}{l|l}
5.0 & 6 \\
\end{tabular} & \begin{tabular}{l|l}
6.2 & 5 \\
\end{tabular} & \begin{tabular}{l|l}
5.5 & 6 \\
\end{tabular} & 6.3 & 15 \\
\hline 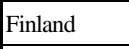 & 5 & 6.0 & 6.6 & 6.3 & 6.6 & 6.7 & 5.6 & 6.0 & 6.7 & 6.4 & 6.7 & 6.6 & 5.5 & 6.1 & 6.7 & 6.3 & 6.6 & 6.7 & 5.56 & 6.16 & 6.76 & $\begin{array}{ll}6.36 \\
\end{array}$ & 6.6 & 6.7 \\
\hline ne & 1 & 0 & 5.3 & 5.3 & 5.7 & 5.4 & \begin{tabular}{|l|}
5.1 \\
\end{tabular} & 4.8 & 5.2 & 5.3 & 5.8 & 5.3 & \begin{tabular}{|l|}
5.1 \\
\end{tabular} & \begin{tabular}{|l|l}
4.8 & \\
\end{tabular} & \begin{tabular}{|l|l|}
5.1 & \\
\end{tabular} & \begin{tabular}{|l|l}
4.9 & 5
\end{tabular} & \begin{tabular}{|l|l}
5.5 \\
\end{tabular} & \begin{tabular}{|l|}
5.3 \\
\end{tabular} & \begin{tabular}{|l|l|}
5.1 & 4 \\
\end{tabular} & \begin{tabular}{l|l}
4.7 & 4 \\
\end{tabular} & \begin{tabular}{l|l}
4.6 & 4 \\
\end{tabular} & \begin{tabular}{l|l}
4.3 & 4 \\
\end{tabular} & 4.9 & 5.3 \\
\hline UK & 4 & 5.3 & 5.1 & 5.3 & 5.9 & 5.7 & 5.5 & 5.4 & 5.2 & 5.3 & 6.0 & 5.9 & 5.4 & \begin{tabular}{|l|l}
5.4 & \\
\end{tabular} & \begin{tabular}{|l|l}
5.2 & 5 \\
\end{tabular} & \begin{tabular}{|l|l}
5.1 & 5 \\
\end{tabular} & \begin{tabular}{|l|l}
5.9 &
\end{tabular} & 5.7 & \begin{tabular}{|l|l|}
5.4 & 5 \\
\end{tabular} & \begin{tabular}{l|l}
5.4 & 5 \\
\end{tabular} & \begin{tabular}{l|l}
5.1 & 5 \\
\end{tabular} & \begin{tabular}{l|l}
5.0 & 5 \\
\end{tabular} & 5.8 & 5.6 \\
\hline & 3.9 & & 5.4 & 4.8 & 5 & ). & 3.9 & 4 & 5.3 & 7 & 5.3 & 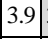 & 3.9 & 3.55 & \begin{tabular}{|l|l}
5.3 & 4 \\
\end{tabular} & 4.75 & 5.4 & 4.0 & 3 & \begin{tabular}{l|l}
3.6 & 5 \\
\end{tabular} & \begin{tabular}{l|l}
5.3 & 4 \\
\end{tabular} & \begin{tabular}{l|l}
4.9 & 5 \\
\end{tabular} & 5 & 4.4 \\
\hline Croatia & $\pi$ & 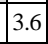 & 6.2 & 5.2 & 4.9 & 4.7 & \begin{tabular}{|l|}
4.0 \\
\end{tabular} & 3.5 & 6.2 & 5.2 & \begin{tabular}{|l|l}
5.2 \\
\end{tabular} & 4.7 & 4.1 & \begin{tabular}{|l|l}
3.6 & 6 \\
\end{tabular} & 6.45 & \begin{tabular}{|l|l}
5.3 & 5 \\
\end{tabular} & \begin{tabular}{|l|l}
5.5 & \\
\end{tabular} & \begin{tabular}{|l|l}
4.6 \\
\end{tabular} & \begin{tabular}{|l|l}
4.1 & 3 \\
\end{tabular} & \begin{tabular}{l|l}
3.6 & 6 \\
\end{tabular} & \begin{tabular}{l|l}
6.5 & 5 \\
\end{tabular} & \begin{tabular}{l|l}
5.2 & 5 \\
\end{tabular} & 5.4 & 4.4 \\
\hline ingary & 4 & 3.8 & 6.4 & 4.9 & 5.4 & 4.2 & 4.3 & 3.7 & 6.5 & 5.0 & 5.4 & 4.2 & \begin{tabular}{|l|}
4.3 \\
\end{tabular} & \begin{tabular}{|l|l}
3.7 & $\mathrm{C}$ \\
\end{tabular} & \begin{tabular}{|l|l|}
6.4 & \\
\end{tabular} & \begin{tabular}{|l|l}
4.9 & 5 \\
\end{tabular} & \begin{tabular}{|l|l|}
5.1 \\
\end{tabular} & \begin{tabular}{|l|}
4.2 \\
\end{tabular} & \begin{tabular}{|l|l|}
4.3 & 3 \\
\end{tabular} & \begin{tabular}{l|l}
3.7 & 6
\end{tabular} & \begin{tabular}{l|l}
6.2 & 4 \\
\end{tabular} & \begin{tabular}{l|l}
4.8 & 4
\end{tabular} & 4.9 & 4.1 \\
\hline Ireland & t & 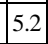 & 6.2 & 5.7 & 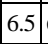 & 0 & \begin{tabular}{|l|l}
4.9 &
\end{tabular} & 5.2 & 6.3 & 5.6 & \begin{tabular}{|l|l|}
6.3 \\
\end{tabular} & 0 & \begin{tabular}{|l|}
4.9 \\
.
\end{tabular} & \begin{tabular}{|l|l|}
5.3 & 6 \\
\end{tabular} & \begin{tabular}{|l|l}
6.1 & \\
\end{tabular} & \begin{tabular}{|l|l}
5.5 & \\
\end{tabular} & \begin{tabular}{|l|l}
5.9 & 0 \\
\end{tabular} & $\begin{array}{ll}6.1 \\
\end{array}$ & $\begin{array}{ll}505 \\
\end{array}$ & \begin{tabular}{l|l}
5.4 & 6 \\
\end{tabular} & \begin{tabular}{l|l}
6.1 & 5 \\
\end{tabular} & \begin{tabular}{l|l}
5.5 & 5 \\
\end{tabular} & 5.9 & 6.1 \\
\hline & & & 5.5 & 4.5 & 3.5 & 5.1 & 4.5 & 6 & 5.6 & 4.5 & 3.5 & 5.1 & 4.4 & 3 & 5.74 & 4.5 & $5: 5$ & 5.0 & 4.4 & \begin{tabular}{l|l}
3.4 & 5
\end{tabular} & \begin{tabular}{l|l}
5.6 & 4
\end{tabular} & \begin{tabular}{l|l}
4.3 & 3
\end{tabular} & 3 & \\
\hline hu & 4 & 3.9 & 6.4 & 5.4 & 5.7 & 4.2 & 4.4 & 4.0 & 6.3 & 5.3 & 5.7 & 4.3 & 4.4 & 4.06 & \begin{tabular}{l|l}
6.3 & 5 \\
\end{tabular} & \begin{tabular}{|l|l}
5.15 \\
\end{tabular} & 5.5 & \begin{tabular}{|l|}
4.2 \\
\end{tabular} & \begin{tabular}{|l|l}
4.5 & 4 \\
\end{tabular} & \begin{tabular}{l|l}
4.0 & 5 \\
\end{tabular} & \begin{tabular}{l|l}
5.7 & 4 \\
\end{tabular} & \begin{tabular}{l|l}
4.8 & 5 \\
\end{tabular} & 5.1 & 4.3 \\
\hline Luxembc & 5 & & \begin{tabular}{|l|}
6.2 \\
\end{tabular} & 6.0 & 6.7 & 5.9 & 5.15 & 5 & 6.2 & 6.2 & 6.8 & 5.9 & 5.1 & \begin{tabular}{|l|l|}
5.6 \\
\end{tabular} & 6.30 & 6.3 & 6.5 & 6.0 & $3.5^{3}$ & \begin{tabular}{l|l}
5.7 & 6 \\
\end{tabular} & \begin{tabular}{l|l}
6.2 & 6 \\
\end{tabular} & \begin{tabular}{l|l}
6.1 & 6 \\
\end{tabular} & 0.3 & 0 \\
\hline & & & 5.9 & 5.2 & 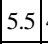 & 4.2 & 4.4 & 4.0 & 6.0 & 5.3 & 5.7 & 4.3 & 4.4 & 4.16 & 6.25 & 5.45 & 5.7 & 4.4 & 4 & 5 & \begin{tabular}{l|l}
5.9 & 5 \\
\end{tabular} & \begin{tabular}{l|l}
5.2 & 5 \\
\end{tabular} & 5 & 4.6 \\
\hline Malta & 3 & 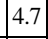 & 6.1 & 6.0 & 6.6 & 5.2 & 4.4 & 4.6 & 5.9 & 5.9 & 6.4 & 5.0 & 4.5 & 4.65 & 5.8 .5 & 5.86 & 6.05 & 5.3 & 54 & \begin{tabular}{l|l}
4.5 & 5 \\
\end{tabular} & \begin{tabular}{l|l}
5.6 & 5 \\
\end{tabular} & \begin{tabular}{l|l}
5.7 & 5 \\
\end{tabular} & 5.8 & 5 \\
\hline Nethe & 4 & 5.6 & 5.8 & 5.2 & 6.1 & 6.1 & $5.5:$ & 5.7 & 6.1 & 5.6 & 6.3 & 6.2 & 5.4 & 5.66 & 6.15 & \begin{tabular}{l|l}
5.5 & 6 \\
\end{tabular} & 6.26 & 6.1 & \begin{tabular}{|l|l|}
5.5 & 5 \\
\end{tabular} & \begin{tabular}{l|l}
5.5 & 5 \\
\end{tabular} & \begin{tabular}{l|l}
5.7 & 5 \\
\end{tabular} & \begin{tabular}{l|l}
5.2 & 6 \\
\end{tabular} & 6.0 & 6.0 \\
\hline an & 5 & 4.2 & 6.0 & 5.5 & 5.7 & 4.4 & 4.5 & 4.1 & 6.1 & 5.5 & \begin{tabular}{|l|}
5.7 \\
\end{tabular} & 4.3 & 4.5 & \begin{tabular}{|l|l|}
4.0 & 6 \\
\end{tabular} & \begin{tabular}{l|l}
6.2 & 5 \\
\end{tabular} & \begin{tabular}{|l|l}
5.4 & 5 \\
\end{tabular} & \begin{tabular}{|l|l}
5.7 \\
\end{tabular} & 4.1 & \begin{tabular}{|l|l|}
4.5 & 4 \\
\end{tabular} & \begin{tabular}{l|l|}
4.0 & 6 \\
\end{tabular} & \begin{tabular}{l|l}
6.0 & 5 \\
\end{tabular} & \begin{tabular}{l|l}
5.4 & 5 \\
\end{tabular} & 5.6 & 4.1 \\
\hline Portugal & & 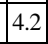 & 6.2 & 5.8 & . & 0 & 4.4 & 4.3 & 6.3 & 5.9 & \begin{tabular}{|l|}
6.2 \\
\end{tabular} & 2 & \begin{tabular}{|l|}
4.4 \\
\end{tabular} & \begin{tabular}{|l|l|}
4.3 & 6 \\
\end{tabular} & 6.5 & \begin{tabular}{|l|l}
5.9 & 6 \\
\end{tabular} & \begin{tabular}{|l|l}
6.2 & \\
\end{tabular} & \begin{tabular}{|l|l}
5.2 \\
\end{tabular} & $4.54^{4}$ & \begin{tabular}{l|l}
4.4 & 6 \\
\end{tabular} & \begin{tabular}{l|l}
6.5 & 6 \\
\end{tabular} & \begin{tabular}{l|l}
6.0 & 6 \\
\end{tabular} & 6.3 & 5.3 \\
\hline Romanıa & 1 & 3.5 & 5.7 & 4.9 & 4.9 & 3.7 & 4.1 & 3.3 & 5.7 & 5.1 & \begin{tabular}{|l|}
4.6 \\
\end{tabular} & 3.4 & 4.1 & \begin{tabular}{|l|l}
3.3 & \\
\end{tabular} & \begin{tabular}{l|l}
5.6 & \\
\end{tabular} & \begin{tabular}{|l|l|}
5.1 & 4 \\
\end{tabular} & 4.73 & 3.6 & \begin{tabular}{|l|l|}
4.3 & 3 \\
\end{tabular} & \begin{tabular}{l|l}
3.6 & 4 \\
\end{tabular} & \begin{tabular}{l|l}
4.8 & 4 \\
\end{tabular} & \begin{tabular}{l|l}
4.4 & 4 \\
\end{tabular} & 4.1 & 4.2 \\
\hline Slovak Rep. & 2 & 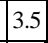 & 6.3 & 5.0 & 4.7 & 3.8 & 4.13 & 3.4 & 6.2 & 4.9 & 4.6 & 30 & 4.1 & $3.3 \mathrm{C}$ & \begin{tabular}{l|l}
6.2 & 4 \\
\end{tabular} & 4.7 & 4.5 & 3.6 & \begin{tabular}{l|l|}
4.2 & 3 \\
\end{tabular} & \begin{tabular}{l|l}
3.3 & 5 \\
\end{tabular} & \begin{tabular}{l|l}
5.9 & 4 \\
\end{tabular} & \begin{tabular}{l|l}
4.5 & 4 \\
\end{tabular} & 46 & 36 \\
\hline sloventa & 3 & 4.1 & & 6.1 & 5.8 & 4.4 & 4.3 & 4.0 & 6.7 & 6.0 & 5.8 & 4.7 & 4.3 & 3.96 & 6.7 & 5.85 & 5.7 & 5.0 & \begin{tabular}{|l|l|}
4.2 & 3 \\
\end{tabular} & \begin{tabular}{l|l}
3.8 & 6
\end{tabular} & \begin{tabular}{l|l}
6.6 & 5 \\
\end{tabular} & \begin{tabular}{l|l}
5.5 & 5 \\
\end{tabular} & 5.5 & 4.9 \\
\hline Sweden & & & 6.3 & 6.0 & 6.6 & 6.0 & 5.5 & 5.7 & 6.2 & 5.7 & 6.0 & 6.1 & 5.5 & 5.76 & 6.1 & 5.56 & 6.0 & 5.9 & 3.43 & \begin{tabular}{l|l|l}
5.4 & 5 \\
\end{tabular} & \begin{tabular}{l|l}
5.8 & 5 \\
\end{tabular} & \begin{tabular}{l|l}
5.2 & 5 \\
\end{tabular} & 5.0 & 5.1 \\
\hline Lu & & 4.6 & 6.0 & 5.4 & 5.8 & 5.1 & 4.7 & 4.5 & 6.0 & 5.4 & - & 5.1 & 4.7 & $4.5 \mathrm{C}$ & 6.05 & 3.5 & 5.65 & $0.1]$ & $4.1]^{4}$ & $\left.4.5\right|^{3}$ & \begin{tabular}{l|l}
5.8 & 5
\end{tabular} & \begin{tabular}{l|l}
5.2 & 5
\end{tabular} & J.J & 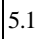 \\
\hline erbia & 3.9 & 3.2 & 5.5 & 4.5 & 4.3 & 3.9 & 3.9 & 3.2 & 5.6 & 4.6 & 4.1 & 4.0 & \begin{tabular}{|l|l}
3.8 \\
\end{tabular} & 3.25 & \begin{tabular}{l|l}
5.6 \\
\end{tabular} & \begin{tabular}{|l|l}
4.3 & 4 \\
\end{tabular} & 4.0 & \begin{tabular}{|l|}
4.0 \\
\end{tabular} & \begin{tabular}{|l|l|}
3.9 & 3 \\
\end{tabular} & \begin{tabular}{l|l}
3.2 & 5
\end{tabular} & \begin{tabular}{l|l}
5.5 & 4
\end{tabular} & \begin{tabular}{l|l}
4.2 & 4
\end{tabular} & 4.1 & \\
\hline
\end{tabular}


Table 1. Security indicators for the EU countries and Serbia in the period 2011-2017 (continued)

\begin{tabular}{|c|c|c|c|c|c|c|c|c|c|c|c|c|c|c|c|c|c|c|}
\hline & & & & 115 & & & & & $\overline{201}$ & & & & & & & 117 & & \\
\hline untry & 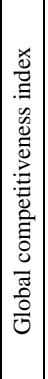 & 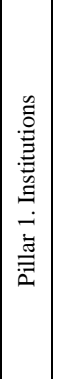 & 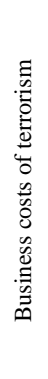 & 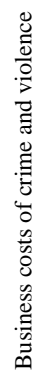 & 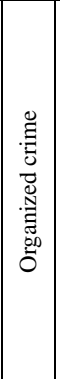 & 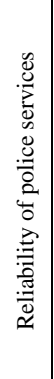 & 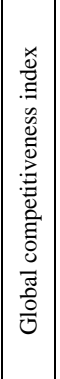 & 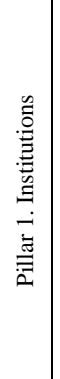 & 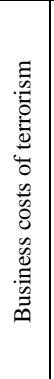 & 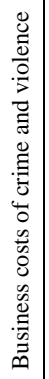 & 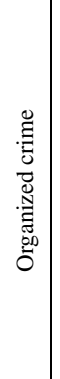 & 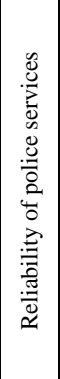 & 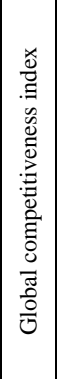 & 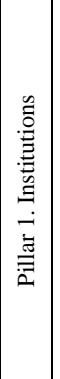 & 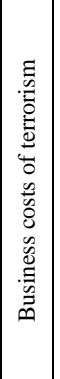 & 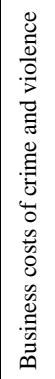 & 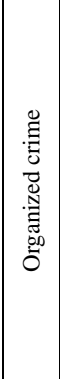 & 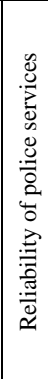 \\
\hline Austria & 5.1 & 5.2 & 6.3 & 6.0 & 6.4 & 5.9 & 5.2 & 5.2 & 5.8 & 5.5 & 6.0 & 6.2 & 5.2 & 5.2 & 5.6 & 5.3 & 5.7 & 6.1 \\
\hline Belgium & 5.2 & 5.2 & 5.7 & 5.4 & 5.8 & 5.7 & 5.3 & \begin{tabular}{|l|}
5.2 \\
\end{tabular} & 4.8 & 5.1 & \begin{tabular}{|l|}
5.4 \\
\end{tabular} & 5.9 & 5.2 & 5.0 & 4.2 & 4.8 & 5.3 & 5.6 \\
\hline Bulgaria & 4.3 & 3.4 & 4.8 & 4.2 & 3.9 & 3.3 & 4.4 & 3.5 & 4.5 & 4.0 & 3.7 & 3.5 & 4.5 & 3.5 & 4.5 & 3.9 & 3.7 & 3.6 \\
\hline Cyprus & 4.2 & 4.3 & 6.0 & 5.8 & 5.6 & 4.7 & 4.0 & 4.0 & 5.5 & 5.3 & \begin{tabular}{|l|}
5.1 \\
\end{tabular} & 4.5 & 4.3 & 4.2 & 5.3 & 5.1 & 4.8 & 4.7 \\
\hline Czech Rep. & 4.7 & 4.1 & 6.0 & 5.2 & 5.6 & 4.1 & 4.7 & 4.2 & 6.0 & 5.3 & 5.7 & 4.5 & 4.8 & 4.2 & 6.0 & 5.5 & 5.9 & 4.8 \\
\hline Germany & 5.5 & 5.2 & 5.1 & 5.0 & 5.3 & 5.9 & \begin{tabular}{|l|}
5.6 \\
\end{tabular} & 5.2 & 4.9 & 4.8 & 5.0 & 5.3 & 5.7 & 5.3 & 5.1 & 5.0 & 5.0 & 5.3 \\
\hline Denmark & 5.3 & 5.5 & 5.0 & 5.3 & 5.8 & 6.0 & 5.3 & 5.5 & 5.1 & 5.4 & 5.8 & 6.0 & 5.4 & 5.5 & \begin{tabular}{|l|l|}
4.9 \\
\end{tabular} & 5.2 & 5.4 & 5.8 \\
\hline Spain & 4.6 & \begin{tabular}{|l|l|}
3.9 \\
\end{tabular} & 5.2 & 5.2 & 5.6 & 5.8 & 4.7 & 4.1 & 5.4 & 5.4 & \begin{tabular}{|l|l|}
5.7 \\
\end{tabular} & 6.2 & \begin{tabular}{|l|}
4.7 \\
\end{tabular} & 4.1 & \begin{tabular}{|l|}
5.3 \\
\end{tabular} & 5.3 & 5.5 & 6.2 \\
\hline Estonia & 4.7 & \begin{tabular}{|l|l|}
5.0 \\
\end{tabular} & 6.2 & 5.5 & 6.4 & 5.3 & 4.8 & 5.1 & 6.2 & 5.5 & 6.3 & 6.1 & 4.8 & 5.0 & 6.1 & 5.6 & 6.2 & 6.0 \\
\hline Finland & 5.5 & 6.1 & 6.7 & 6.5 & 6.8 & 6.7 & 5.4 & 6.1 & 6.4 & 6.4 & 6.7 & 6.8 & 5.5 & 6.2 & 6.5 & 6.3 & 6.8 & 6.8 \\
\hline France & 5.1 & \begin{tabular}{|l|l}
4.8 \\
\end{tabular} & 4.5 & 4.5 & 5.0 & 5.3 & 5.2 & 4.9 & 4.3 & 4.8 & \begin{tabular}{|l|}
5.1 \\
\end{tabular} & 5.8 & 5.2 & 4.8 & 4.2 & 4.9 & 5.1 & 5.7 \\
\hline UK & 5.4 & 5.5 & 5.1 & 5.2 & 5.7 & 5.6 & 5.5 & 5.5 & 4.8 & 5.1 & \begin{tabular}{|l|l}
5.6 \\
\end{tabular} & 6.1 & 5.5 & 5.5 & 4.7 & 5.0 & 5.5 & 6.0 \\
\hline Greece & 4.0 & \begin{tabular}{|l|l}
3.7 \\
\end{tabular} & 5.4 & 5.0 & 5.3 & 4.4 & 4.0 & 3.8 & 5.4 & 5.0 & \begin{tabular}{|l|l|}
5.3 \\
\end{tabular} & 4.7 & 4.0 & \begin{tabular}{|l|}
3.7 \\
\end{tabular} & \begin{tabular}{|l|}
5.3 \\
\end{tabular} & 4.9 & 5.1 & 4.4 \\
\hline Croatia & 4.1 & 3.6 & 6.4 & 5.4 & 5.3 & 4.4 & 4.1 & 3.6 & 6.2 & 5.6 & \begin{tabular}{|l|l|}
5.1 \\
\end{tabular} & 4.9 & 4.2 & 3.5 & 5.9 & 5.3 & 4.9 & 4.6 \\
\hline Hungary & 4.3 & 3.5 & 6.2 & 5.0 & 4.6 & 4.1 & 4.2 & 3.3 & 4.8 & 5.7 & \begin{tabular}{|l|l}
4.6 \\
\end{tabular} & 4.3 & 4.3 & 3.5 & 5.3 & 5.6 & 5.1 & 4.5 \\
\hline Ireland & 5.1 & 5.5 & 6.3 & 5.5 & 6.1 & 6.1 & 5.2 & 5.6 & 6.1 & 5.2 & \begin{tabular}{|l|l|}
5.8 \\
\end{tabular} & 6.1 & 5.2 & 5.3 & 5.5 & 4.9 & 5.5 & 5.8 \\
\hline Italy & 4.5 & 3.4 & 5.3 & 4.1 & 3.3 & 4.8 & 4.5 & 3.5 & 5.1 & 4.0 & 3.5 & 4.3 & 4.5 & 3.5 & 4.9 & 4.0 & 3.5 & 4.5 \\
\hline Lithuania & 4.6 & 4.1 & 5.4 & 5.0 & 5.2 & 4.3 & 4.6 & 4.2 & 5.6 & 5.1 & \begin{tabular}{|l|}
5.4 \\
\end{tabular} & 4.7 & 4.6 & 4.1 & 5.6 & 5.1 & 5.5 & \begin{tabular}{|l|l}
4.7 \\
\end{tabular} \\
\hline Luxembourg & 5.2 & 5.8 & 6.1 & 6.0 & 6.2 & 6.1 & 5.2 & \begin{tabular}{|l|}
5.8 \\
\end{tabular} & 5.7 & 5.8 & 6.1 & 6.2 & 5.2 & 5.7 & 5.6 & 5.9 & 6.2 & 6.2 \\
\hline Latvia & 4.5 & \begin{tabular}{|l|l|}
4.2 \\
\end{tabular} & 6.1 & 5.3 & 6.1 & 4.6 & 4.4 & 4.0 & 5.9 & 5.3 & \begin{tabular}{|l|l|}
5.8 \\
\end{tabular} & 4.2 & 4.4 & 3.8 & 6.1 & 5.2 & 5.5 & 4.3 \\
\hline Malta & 4.4 & 4.5 & 5.7 & 5.7 & 6.0 & 5.3 & 4.5 & 4.5 & 5.4 & 5.4 & 5.7 & 5.0 & 4.6 & 4.5 & 5.5 & 5.5 & 5.7 & 4.8 \\
\hline Netherlands & 5.5 & 5.6 & 5.5 & 5.2 & 5.9 & 6.0 & 5.6 & 5.7 & 5.4 & 5.2 & \begin{tabular}{|l|l}
5.8 \\
\end{tabular} & 6.2 & 5.7 & 5.8 & 5.2 & 5.1 & 5.7 & 6.1 \\
\hline Poland & 4.5 & 4.1 & 5.7 & 5.2 & 5.4 & 4.1 & 4.6 & 4.0 & 5.5 & 5.0 & \begin{tabular}{|l|l|}
5.2 \\
\end{tabular} & 4.1 & 4.6 & 3.8 & 5.3 & 4.9 & 5.1 & 4.1 \\
\hline Portugal & 4.5 & 4.4 & 6.3 & 6.0 & 6.3 & 5.3 & 4.5 & 4.3 & 6.1 & 5.9 & \begin{tabular}{|l|l|}
6.2 & \\
\end{tabular} & 5.7 & 4.6 & 4.4 & 6.0 & 5.8 & 6.0 & 5.7 \\
\hline Romania & 4.3 & \begin{tabular}{|l|l}
3.7 \\
\end{tabular} & 5.2 & 4.9 & 4.6 & 4.2 & 4.3 & 3.6 & 5.7 & 5.3 & \begin{tabular}{|l|l|}
5.1 \\
\end{tabular} & 4.2 & \begin{tabular}{|l|l|}
4.3 \\
\end{tabular} & \begin{tabular}{|l|}
3.7 \\
\end{tabular} & \begin{tabular}{|l|}
5.3 \\
\end{tabular} & 5.1 & 4.8 & 4.4 \\
\hline Slovak Rep. & 4.2 & 3.4 & 5.8 & 4.8 & 4.8 & 3.6 & 4.3 & 3.5 & 5.8 & 4.8 & \begin{tabular}{|l|l}
4.9 \\
\end{tabular} & 3.6 & 4.3 & 3.5 & 5.8 & 4.8 & 5.0 & 3.5 \\
\hline Slovenia & 4.3 & \begin{tabular}{|l|l}
3.9 \\
\end{tabular} & 6.3 & 5.6 & 5.5 & 4.9 & 4.4 & 4.1 & 5.9 & 5.8 & \begin{tabular}{|l|l|}
5.7 \\
\end{tabular} & 5.5 & 4.5 & \begin{tabular}{|l|l}
4.1 \\
\end{tabular} & 5.6 & 5.6 & 5.4 & 5.3 \\
\hline Sweden & 5.4 & 5.6 & 5.8 & 5.4 & 5.7 & 5.7 & 5.5 & \begin{tabular}{|l|}
5.9 \\
\end{tabular} & 6.0 & 5.8 & \begin{tabular}{|l|l|}
6.2 \\
\end{tabular} & 5.7 & 5.5 & 5.6 & 5.5 & 5.2 & 5.6 & 5.3 \\
\hline $\mathrm{EU}$ & 4.8 & 4.5 & 5.7 & 5.3 & 5.5 & 5.1 & 4.8 & 4.6 & 5.5 & 5.3 & \begin{tabular}{|l|l|}
5.4 & \\
\end{tabular} & 5.2 & 4.8 & 4.5 & 5.4 & 5.2 & 5.3 & 5.2 \\
\hline Serbia & 3.9 & 3.2 & 5.4 & 4.4 & 4.3 & 3.8 & 4.0 & 3.3 & 5.1 & 4.4 & 4.1 & 3.7 & 4.1 & 3.4 & 5.0 & 4.4 & 4.1 & 3.9 \\
\hline
\end{tabular}


b) The analysis of interdependence between national security indicators and the Global Competitiveness Index of the EU countries and Serbia

The interdependence of national security indicators can be determined using correlation analysis. Namely, the Pearson's correlation coefficient, as a measure of the linear relationship between indicators, represents a range of values from 0 to 1 indicating the strength of their correlation (Soldić-Aleksić, 2015, p. 177):

$$
r=\frac{\operatorname{cov}_{x z}}{s_{x} s_{y}}=\frac{\sum\left(x_{i}-\bar{x}\right)\left(y_{i}-\bar{y}\right)}{(n-1) s_{x} s_{y}}
$$

For the values of the Pearson's correlation coefficient between 0.10 and 0.29 , the correlation is considered to be low; if the Pearson's correlation coefficient falls between 0.30 and 0.49 , the correlation is medium, and the correlation is high if the Pearson's correlation coefficient scores above 0.50 (Soldić-Aleksić, 2015, p. 180).

The relationship between variables can be both positive and negative. If variables change in the same direction, precisely, if a direction change of one variable follows the change of other variable(s) in the same direction, the relationship is positive. On the other hand, the relationship is considered to be a negative one if variables change in opposite directions. However, before the correlation analysis is applied, it is of paramount to investigate the existence of the relationship between indicators, based on the concept of statistical significance.

The coefficient of determination, as a squared Pearson's coefficient of correlation $\mathrm{R}^{2}$, can be also used for the purpose of data interpretation (table 2). Namely, the coefficient of determination shows the common variance of two variables, or how much of the variance of one variable is explained and caused by the variance of another variable (Soldic-Aleksic, 2015, p. 180).

Table 2 illustrates the results of correlation analysis between GCI and four security indicators, as well as the correlation with the first GCI pillar Institutions, for the analyzed countries over the period 2011-2017.

Table 2. Correlation analysis of the security indicators and national competitiveness, for the EU countries and Serbia, 2011-2017

\begin{tabular}{crrr}
\hline \multicolumn{1}{c}{ Correlation } & $\begin{array}{c}\text { Pearson } \\
\text { Correlation }\end{array}$ & $\begin{array}{c}\text { Coefficient of } \\
\text { determination }\end{array}$ & $\begin{array}{c}\text { *Sig. } \\
\text { (2-tailed) }\end{array}$ \\
\hline Austria: GCI-Pillar 1_Institutions & -0.496 & $24.60 \%$ & 0.257 \\
GCI-Business costs of terrorism & -0.216 & $4.67 \%$ & 0.642 \\
GCI-Business costs of crime and violence & -0.098 & $0.96 \%$ & 0.835 \\
GCI-Organized crime & -0.289 & $8.35 \%$ & 0.530 \\
GCI-Reliability of police services & 0.482 & $23.23 \%$ & 0.273 \\
\hline Belgium: GCI-Pillar 1_Institutions & 0.607 & $36.84 \%$ & 0.148 \\
GCI-Business costs of terrorism & -0.475 & $22.56 \%$ & 0.281 \\
GCI-Business costs of crime and violence & -0.333 & $11.09 \%$ & 0.465 \\
GCI-Organized crime & -0.458 & $20.98 \%$ & 0.301 \\
GCI-Reliability of police services & 0.810 & $65.61 \%$ & 0.027 \\
\hline
\end{tabular}




\begin{tabular}{|c|c|c|c|}
\hline Bulgaria: GCI-Pillar 1_Institutions & 0.627 & $39.31 \%$ & 0.131 \\
\hline GCI-Business costs of terrorism & -0.512 & $26.21 \%$ & 0.240 \\
\hline GCI-Business costs of crime and violence & 0.055 & $0.30 \%$ & 0.907 \\
\hline GCI-Organized crime & -0.495 & $24.50 \%$ & 0.259 \\
\hline GCI-Reliability of police services & 0.571 & $32.60 \%$ & 0.181 \\
\hline Cyprus: GCI-Pillar 1_Institutions & 0.842 & $70.90 \%$ & 0.018 \\
\hline GCI-Business costs of terrorism & 0.379 & $14.36 \%$ & 0.402 \\
\hline GCI-Business costs of crime and violence & 0.307 & $9.42 \%$ & 0.503 \\
\hline GCI-Organized crime & 0.390 & $15.21 \%$ & 0.387 \\
\hline GCI-Reliability of police services & 0.763 & $58.22 \%$ & 0.046 \\
\hline Czech GCI-Pillar 1_Institutions & 0.952 & $90.63 \%$ & 0.001 \\
\hline Republic: GCI-Business costs of terrorism & -0.566 & $32.04 \%$ & 0.185 \\
\hline GCI-Business costs of crime and violence & 0.450 & $20.25 \%$ & 0.311 \\
\hline GCI-Organized crime & 0.883 & $77.97 \%$ & 0.008 \\
\hline GCI-Reliability of police services & 0.829 & $68.72 \%$ & 0.021 \\
\hline Germany: GCI-Pillar 1_Institutions & -0.047 & $0.22 \%$ & 0.921 \\
\hline GCI-Business costs of terrorism & -0.712 & $50.69 \%$ & 0.073 \\
\hline GCI-Business costs of crime and violence & -0.632 & $39.94 \%$ & 0.128 \\
\hline GCI-Organized crime & -0.800 & $64.00 \%$ & 0.031 \\
\hline GCI-Reliability of police services & -0.854 & $72.93 \%$ & 0.014 \\
\hline Denmark: GCI-Pillar 1_Institutions & 0.795 & $63.20 \%$ & 0.033 \\
\hline GCI-Business costs of terrorism & 0.494 & $24.40 \%$ & 0.259 \\
\hline GCI-Business costs of crime and violence & 0.723 & $52.27 \%$ & 0.66 \\
\hline GCI-Organized crime & 0.426 & $18.15 \%$ & 0.341 \\
\hline GCI-Reliability of police services & -0.085 & $0.72 \%$ & 0.856 \\
\hline Spain: GCI-Pillar 1_Institutions & -0.243 & $5.90 \%$ & 0.600 \\
\hline GCI-Business costs of terrorism & 0.693 & $48.02 \%$ & 0.085 \\
\hline GCI-Business costs of crime and violence & -0.108 & $1.17 \%$ & 0.817 \\
\hline GCI-Organized crime & -0.304 & $9.24 \%$ & 0.507 \\
\hline GCI-Reliability of police services & 0.857 & $73.44 \%$ & 0.014 \\
\hline Estonia: GCI-Pillar 1_Institutions & 0.592 & $35.05 \%$ & 0.162 \\
\hline GCI-Business costs of terrorism & -0.814 & $66.26 \%$ & 0.026 \\
\hline GCI-Business costs of crime and violence & 0.418 & $17.47 \%$ & 0.350 \\
\hline GCI-Organized crime & -0.935 & $87.42 \%$ & 0.002 \\
\hline GCI-Reliability of police services & 0.660 & $43.56 \%$ & 0.107 \\
\hline Finland: GCI-Pillar 1_Institutions & -0.418 & $17.47 \%$ & 0.350 \\
\hline GCI-Business costs of terrorism & 0.713 & $50.84 \%$ & 0.072 \\
\hline GCI-Business costs of crime and violence & 0.000 & $0.00 \%$ & 1.000 \\
\hline GCI-Organized crime & 0.000 & $0.00 \%$ & 1.000 \\
\hline GCI-Reliability of police services & -0.837 & $70.06 \%$ & 0.019 \\
\hline France: GCI-Pillar 1_Institutions & 0.154 & $2.37 \%$ & 0.742 \\
\hline GCI-Business costs of terrorism & -0.748 & $55.95 \%$ & 0.053 \\
\hline GCI-Business costs of crime and violence & -0.013 & $0.02 \%$ & 0.978 \\
\hline GCI-Organized crime & -0.379 & $14.36 \%$ & 0.402 \\
\hline GCI-Reliability of police services & 0.976 & $95.26 \%$ & 0.000 \\
\hline UK: GCI-Pillar 1_Institutions & 0.471 & $22.18 \%$ & 0.286 \\
\hline GCI-Business costs of terrorism & -0.609 & $37.09 \%$ & 0.147 \\
\hline GCI-Business costs of crime and violence & -0.070 & $0.49 \%$ & 0.881 \\
\hline GCI-Organized crime & -0.371 & $13.76 \%$ & 0.412 \\
\hline GCI-Reliability of police services & 0.935 & $87.42 \%$ & 0.002 \\
\hline Greece: GCI-Pillar 1_Institutions & 0.882 & $77.79 \%$ & 0.009 \\
\hline GCI-Business costs of terrorism & 0.167 & $2.79 \%$ & 0.721 \\
\hline GCI-Business costs of crime and violence & 0.910 & $82.81 \%$ & 0.004 \\
\hline GCI-Organized crime & -0.383 & $14.67 \%$ & 0.397 \\
\hline GCI-Reliability of police services & 0.926 & $85.75 \%$ & 0.003 \\
\hline
\end{tabular}




\begin{tabular}{|c|c|c|c|c|}
\hline Croatia: & GCI-Pillar 1_Institutions & 0.000 & $0.00 \%$ & 1.000 \\
\hline & GCI-Business costs of terrorism & -0.436 & $19.01 \%$ & 0.329 \\
\hline & GCI-Business costs of crime and violence & 0.197 & $3.88 \%$ & 0.672 \\
\hline & GCI-Organized crime & -0.370 & $13.69 \%$ & 0.414 \\
\hline & GCI-Reliability of police services & -0.163 & $2.66 \%$ & 0.727 \\
\hline Hungary: & GCI-Pillar 1_Institutions & 0.833 & $69.39 \%$ & 0.020 \\
\hline & GCI-Business costs of terrorism & 0.705 & $49.70 \%$ & 0.077 \\
\hline & GCI-Business costs of crime and violence & -0.635 & $40.32 \%$ & 0.126 \\
\hline & GCI-Organized crime & 0.692 & $47.89 \%$ & 0.085 \\
\hline & GCI-Reliability of police services & -0.209 & $4.37 \%$ & 0.653 \\
\hline Ireland: & GCI-Pillar 1_Institutions & 0.731 & $53.44 \%$ & 0.062 \\
\hline & GCI-Business costs of terrorism & -0.537 & $28.84 \%$ & 0.214 \\
\hline & GCI-Business costs of crime and violence & -0.858 & $73.62 \%$ & 0.014 \\
\hline & GCI-Organized crime & -0.798 & $63.68 \%$ & 0.031 \\
\hline & GCI-Reliability of police services & -0.218 & $4.75 \%$ & 0.639 \\
\hline Italy: & GCI-Pillar 1_Institutions & 0.000 & $0.00 \%$ & 1.000 \\
\hline & GCI-Business costs of terrorism & -0.675 & $45.56 \%$ & 0.096 \\
\hline & GCI-Business costs of crime and violence & -0.642 & $41.22 \%$ & 0.120 \\
\hline & GCI-Organized crime & -0.079 & $0.62 \%$ & 0.867 \\
\hline & GCI-Reliability of police services & -0.510 & $26.01 \%$ & 0.242 \\
\hline Lithuania: & GCI-Pillar 1_Institutions & 0.854 & $72.93 \%$ & $\overline{0.014}$ \\
\hline & GCI-Business costs of terrorism & -0.961 & $92.35 \%$ & 0.001 \\
\hline & GCI-Business costs of crime and violence & -0.512 & $26.21 \%$ & 0.240 \\
\hline & GCI-Organized crime & -0.580 & $33.64 \%$ & 0.172 \\
\hline & GCI-Reliability of police services & 0.760 & $57.76 \%$ & 0.047 \\
\hline Luxemburg: & GCI-Pillar 1_Institutions & 0.519 & $26.94 \%$ & 0.233 \\
\hline & GCI-Business costs of terrorism & -0.559 & $31.25 \%$ & 0.192 \\
\hline & GCI-Business costs of crime and violence & -0.405 & $16.40 \%$ & 0.367 \\
\hline & GCI-Organized crime & -0.860 & $73.96 \%$ & 0.013 \\
\hline & GCI-Reliability of police services & 0.880 & $77.44 \%$ & 0.009 \\
\hline Latvia: & GCI-Pillar 1_Institutions & 0.619 & $38.32 \%$ & 0.138 \\
\hline & GCI-Business costs of terrorism & 0.274 & $7.51 \%$ & 0.552 \\
\hline & GCI-Business costs of crime and violence & 0.220 & $4.84 \%$ & 0.635 \\
\hline & GCI-Organized crime & 0.655 & $42.90 \%$ & 0.110 \\
\hline & GCI-Reliability of police services & 0.782 & $61.15 \%$ & 0.038 \\
\hline Malta: & GCI-Pillar 1_Institutions & -0.713 & $50.84 \%$ & 0.072 \\
\hline & GCI-Business costs of terrorism & -0.820 & $67.24 \%$ & 0.024 \\
\hline & GCI-Business costs of crime and violence & -0.773 & $59.75 \%$ & 0.042 \\
\hline & GCI-Organized crime & -0.886 & $78.50 \%$ & 0.008 \\
\hline & GCI-Reliability of police services & -0.444 & $19.71 \%$ & 0.318 \\
\hline Netherlands: & GCI-Pillar 1_Institutions & 0.730 & $53.29 \%$ & 0.062 \\
\hline & GCI-Business costs of terrorism & -0.810 & $65.61 \%$ & 0.027 \\
\hline & GCI-Business costs of crime and violence & -0.490 & $24.01 \%$ & 0.265 \\
\hline & GCI-Organized crime & -0.794 & $63.04 \%$ & 0.033 \\
\hline & GCI-Reliability of police services & 0.191 & $3.65 \%$ & 0.682 \\
\hline Poland: & GCI-Pillar 1_Institutions & -0.701 & $49.14 \%$ & 0.080 \\
\hline & GCI-Business costs of terrorism & -0.873 & $76.21 \%$ & 0.010 \\
\hline & GCI-Business costs of crime and violence & -0.904 & $81.72 \%$ & 0.005 \\
\hline & GCI-Organized crime & -0.901 & $81.18 \%$ & 0.006 \\
\hline & GCI-Reliability of police services & -0.389 & $15.13 \%$ & 0.388 \\
\hline Portugal: & GCI-Pillar 1_Institutions & 0.750 & $56.25 \%$ & 0.052 \\
\hline & GCI-Business costs of terrorism & -0.533 & $28.41 \%$ & 0.218 \\
\hline & GCI-Business costs of crime and violence & 0.000 & $0.00 \%$ & 1.000 \\
\hline & GCI-Organized crime & -0.441 & $19.45 \%$ & 0.322 \\
\hline & GCI-Reliability of police services & 0.824 & $67.90 \%$ & 0.023 \\
\hline
\end{tabular}


1212

\begin{tabular}{|c|c|c|c|}
\hline Romania: GCI-Pillar 1_Institutions & 0.889 & $79.03 \%$ & 0.007 \\
\hline GCI-Business costs of terrorism & -0.645 & $41.60 \%$ & 0.117 \\
\hline GCI-Business costs of crime and violence & -0.202 & $4.08 \%$ & 0.664 \\
\hline GCI-Organized crime & -0.142 & $2.02 \%$ & 0.761 \\
\hline GCI-Reliability of police services & 0.955 & $91.20 \%$ & 0.001 \\
\hline Slovak GCI-Pillar 1_Institutions & 0.681 & $46.38 \%$ & 0.092 \\
\hline Republic: GCI-Business costs of terrorism & -0.730 & $53.29 \%$ & 0.062 \\
\hline GCI-Business costs of crime and violence & 0.000 & $0.00 \%$ & 1.000 \\
\hline GCI-Organized crime & 0.907 & $82.26 \%$ & 0.005 \\
\hline GCI-Reliability of police services & -0.584 & $34.11 \%$ & 0.168 \\
\hline Slovenia: GCI-Pillar 1_Institutions & 0.762 & $58.06 \%$ & 0.046 \\
\hline GCI-Business costs of terrorism & -0.853 & $72.76 \%$ & 0.015 \\
\hline GCI-Business costs of crime and violence & -0.034 & $0.12 \%$ & 0.942 \\
\hline GCI-Organized crime & -0.281 & $7.90 \%$ & 0.542 \\
\hline GCI-Reliability of police services & 0.618 & $38.19 \%$ & 0.139 \\
\hline Sweden: GCI-Pillar 1_Institutions & 0.867 & $75.17 \%$ & 0.011 \\
\hline GCI-Business costs of terrorism & 0.575 & $33.06 \%$ & 0.177 \\
\hline GCI-Business costs of crime and violence & 0.747 & $55.80 \%$ & 0.054 \\
\hline GCI-Organized crime & 0.833 & $69.39 \%$ & 0.020 \\
\hline GCI-Reliability of police services & 0.341 & $11.63 \%$ & 0.454 \\
\hline EU: GCI-Pillar 1_Institutions & 0.091 & $0.83 \%$ & 0.846 \\
\hline GCI-Business costs of terrorism & -0.892 & $79.57 \%$ & 0.007 \\
\hline GCI-Business costs of crime and violence & -0.382 & $14.59 \%$ & 0.398 \\
\hline GCI-Organized crime & -0.778 & $60.53 \%$ & 0.040 \\
\hline GCI-Reliability of police services & 0.730 & $53.29 \%$ & 0.062 \\
\hline Serbia: GCI-Pillar 1_Institutions & 0.923 & $85.19 \%$ & 0.003 \\
\hline GCI-Business costs of terrorism & -0.924 & $85.38 \%$ & 0.003 \\
\hline GCI-Business costs of crime and violence & 0.136 & $1.85 \%$ & 0.772 \\
\hline GCI-Organized crime & 0.022 & $0.05 \%$ & 0.963 \\
\hline GCI-Reliability of police services & -0.382 & $14.59 \%$ & 0.397 \\
\hline
\end{tabular}

Based on the correlation analysis results provided in table 2, the EU itself and the majority of member countries have a positive correlation between GCI and Institutions, as well as between GCI and the reliability of police services, over the period 2011-2017. Accordingly, the rise in the efficiency of the institutional environment, in both private and public sectors, leads at the same time to an increase of GCI. Also, higher reliability of police services leads to an increase of GCI as well. The mentioned correlation is the highest in the Czech Republic, Greece and Romania. However, the correlation between GCI on the one side and business costs of terrorism, business costs of crime and violence, and organized crime on the other side, is negative, with some exceptions. Furthermore, a rise in business costs of terrorism, business costs of crime and violence, and organized crime lead to the decrease in the global competitiveness index (GCI). 


\section{CONCLUSION}

A modern understanding of the concept of security has replaced the traditional understanding of security which relies only on military security. This modern concept implies the degree of human development that has been achieved, reflected in the synthesis of the citizens' security and the security of the state, but also their participation in the spheres of international and global security. The state remains the dominant player in all aspects of national security, such as monetary, political, economic, energetic, environmental and that pertaining to natural resources. However, the existence of any of the various security risks can jeopardize the national economy and its competitiveness.

According to the results of the performed empirical analysis, the EU countries and Serbia as a candidate country, experienced fluctuations in the four security indicators (business costs of terrorism, business costs of crime and violence, organized crime, and the reliability of policy services) being analyzed for the period 2011-2017. Namely, the country that constantly has satisfactory values of all four indicators is Finland. On the other hand, Bulgaria is a country with almost the lowest performance regarding security issues.

The conducted correlation analysis results confirm the positive correlation between national competitiveness and institutions (public and private), as well as between national competitiveness and reliability on policy services. On the contrary, the rise in the business costs of terrorism, business costs of crime and violence and organized crime all have a negative impact on the overall national competitiveness.

\section{REFERENCES}

Baldwin, D.A. (1997). The concept of security. Review of International Studies, vol. 23, pp. 5-26.

Borrus, M.\&Zysman, J. (1990). Industrial competitiveness and American national security, UC Berkeley

Botos, K. (2006). Public economies and international competitiveness. Iustum Aequum Salutare, vol. 2, no 1-2, pp. 13-20.

Cho, T-S \& Moon, H-C. (2001). From Adam Smith to Michael Porter - Evolution of Competitiveness Theory. Singapore: World Scientific \& Imperial College Press.

Frane, A. (2014). Measuring National Innovation Performance, Springer Briefs in Economics

Krstić, B., Stanojević, J. \& Stanišić, T. (2016). Innovations as a determinant of competitiveness of Serbia: a comparative analysis with Western Balkan countries and the European Union, Teme, vol. XL, no 3, pp. 1035-1050.

Lang, P. (2009). Theoretical frameworks of national competitiveness, National competitiveness of Vietnam: determinants, emerging key issues and recommendations, pp. 25-44.

Long, C. (2013). An imperfect balance: ITAR exemption, national security and U.S. competitiveness. National Security Law Journal, vol. 2, no. 1, pp. 43-64. 
Mijalković, S. (2009). Nacionalna bezbednost - od Vestfalskog koncepta do post hladnoratovskog. [National Security - from the Westphalian Concept to PostCold War] Nacionalna bezbednost, str. 55-73.

Nikolaevich, M.D., Jur'evna I.F., Alexandrovna L.P., Maratovich T.T., Vladimirovna M.B., Borisovich A.I. (2018). International Practices to Improve Economic Security, European Research Studies Journal, vol. XXI, no 1, pp. 459-467.

Simanavičienè, Ž \& Stankevičius, A. (2015). Economic security and national competitiveness. Public Security and Public Order, no. 15, pp. 126-143.

Skare, M.\&Rabar, D. (2017). Measuring Sources of Economic Growth in OECD, Inzinerine Ekonomika - Engineering Economics, Vol. 28, No. 4, pp. 386-400

Soldić-Aleksić, J. (2015). Primenjena analiza podataka [Applied Data Analysis], Ekonomski fakultet u Beogradu

Stanojević, J. (2018). Strukturne promene u poljoprivredi Republike Srbije u funkciji unapređenja konkurentnosti [Structural changes in agriculture of the Republic of Serbia aimed at improving competitiveness], $\mathrm{PhD}$ Dissertation, Retrieved from http://nardus.mpn.gov.rs/handle/123456789/10409

Strategija nacionalne bezbednosti Republike Srbije [National security strategy of the Republic of Serbia], Beograd, 2009

Strategija nacionalne bezbednosti Republike Srbije - nacrt [National security strategy of the Republic of Serbia - draft], Beograd, 2017

World Economic Forum - WEF (2013). Global Competitiveness Report. Geneva: World Economic Forum,www.worldbank.org

World Economic Forum - WEF (2018). Global Competitiveness Report. Geneva: World Economic Forum, www.worldbank.org.

\title{
БАЛАНСИРАЊЕ НАЦИОНАЛНЕ БЕЗБЕДНОСТИ И КОНКУРЕНТНОСТИ У ДОБА ИНФОРМАЦИЈА
}

\author{
Јелена Станојевић ${ }^{1}$, Милош Павловић ${ }^{2}$ \\ ${ }^{1}$ Универзитет у Приштини, Економски факултет, Косовска Митровица, Србија \\ ${ }^{2}$ Академија пословних струковних студија, Београд, Србија
}

\section{Резиме}

Изузетно брз научни и технолошки развој, широка примена савремених научних и информационих достигнућа и њихов велики утицај на све области друштвеног живота - повећавају сложеност глобалног окружења. Супротно очекивањима да ће се ниво одговорности свих актера повећати са становишта коришћења научног напретка у општем интересу и за добробит целог човечанства, сматра се да ће развој науке и технологије бити подложан различитим облицима злоупотреба и водити до негативних импликација по безбедност. Динамика развоја глобалне информационе технологије додатно ће олакшати и интензивирати различите криминалне активности. Стога се национална и међународна безбедност јављају као један од већих изазова последњих година, али и као главни циљ националних и међународних организација.

У раду се истражује национална конкурентност кроз призму националне безбедности како би се проценила корелација безбедности и конкурентности. Аутори су препознали показатеље националне безбедности који су кључни за националну конкурентност и доприносе позиционирању привреде на међународној сцени. 
Циљ истраживања је сагледавање традиционалног и савременог концепта националне безбедности и њена анализа на основу следећих показатеља: пословни трошкови проузроковани тероризмом, пословни трошкови проузроковани криминалом и насиљем, организовани криминал и поузданост политичких услуга. Четири поменута показатеља припадају првом од дванаест стубова конкурентности који су обухваћени Индексом глобалне конкурентности, а односе се пре свега на државне институције. Квалитет и конкурентност самих институција има утицаја на укупну националну конкурентност и економски раст утичући на инвестиционе одлуке и друге економске активности. Наиме, инвеститори нису спремни да уложе капитал у случају небезбедног окружења и незаштићених права појединаца.

Истраживањем су обухваћене земље Европске уније и Србија као земља кандидат. Информациону базу чине подаци Светског економског форума који су доступни у Извештајима о глобалној конкурентности за период од 2011. до 2017. године. Методе коришћене у анализи су дескриптивна статистика, корелација и компаративна анализа. Сврха анализе је да се испита тренд четири показатеља која одражавају националну безбедност земаља ЕУ и да се испита положај Србије у односу на ЕУ. Такође, корелационом анализом указује се на однос између националне безбедности и конкурентности како би се пре свега утврдио утицај који безбедност има на националну конкурентност.

Према резултатима спроведене емпиријске анализе, земље ЕУ и Србија, као земља кандидат, доживеле су извесна варирања у четири анализирана показатеља безбедности. Земља ЕУ која константно има задовољавајуће вредности сва четири показатеља у анализираном периоду јесте Финска. Са друге стране, Бугарска је земља са готово најнижим перформансама у погледу безбедности. Србија је у анализираном периоду и у дата четири показатеља безбедности остварила вредности које не одступају много у односу на ЕУ. Међутим, са становишта првог стуба конкурентности - Институције, Србија остварује резултате који су благо испод резултата које остварују земље ЕУ.

Резултати корелационе корекције потврдили су позитивну корелацију између националне конкурентности и институција (јавних и приватних), као и између националне конкурентности и поузданости политичких услуга. Са друге стране, раст пословних трошкова проузрокованих тероризмом, пословних трошкова проузрокованих криминалом и насиљем, те раст организованог криминала - негативно утичу на укупну националну конкурентност. 